\title{
La perfectibilité hybride, Vers une autosanté inhumaine ou citoyenne?
}

\author{
« Je m'invente aujourd'hui des chemins de traverse, \\ Je ne suis plus de chez vous, j'attends des mutants \\ Biologiquement, \\ Je me m'arrange avec l'idée que je me fais de la biologie»"
}

Léo Ferré, La solitude, 1972.

\section{Une nouvelle perfectibilité}

Entre la déconstruction du corps naturel et la cyborgisation du posthumanisme, l'hybridation bio-technologique est une voie moyenne et classique depuis les Métamorphoses d'Ovide, au moins. La déconstruction de la catégorie monstre par les disabilities studies a rendu nécessaire la critique du handicap comme limitation de l'humain. Non que, ce que Catherine Malabou appelle les accidents de plasticité ${ }^{1}$ n'existeraient plus, la condition incarnée du corps suffirait à le rappeler. Le corps humain, même si les limites de la mort seront encore reculées ${ }^{2}$, engage le sujet dans le vécu de son vivant. Corriger les difformités de la nature est devenu une possibilité même de sélectionner les nouveaux nés ou, ce qui est très différent, de réparer par la chirurgie, même du visage les accidents corporels. Comme l'indique Jacques Testart, " la pulsion d'accaparement du plus petit de notre espèce pourrait ainsi relever d'une exigence de consommation cannibale $»^{3}$

La réparation du corps modifie son fonctionnement et le vécu du sujet. Les progrès des prothèses, implants et nanobiologie définissent un corps démonstruisé mais remastérisé par des compléments de soi qui procure une nouvelle clôture d'un moi peau reconstitué. La technique fait performer le corps dans l'actualisation de potentialités inexploités jusque là, comme les cellules souches ${ }^{4}$, ou dans la complémentation de procédés technologiques qui prolongent la vie et ses conditions d'exercices. L'obstacle de la dégénérescence immunitaire ou nerveuse exige que la technique pense encore davantage l'interaction entre le médicament et le vivant comme l'atteste la nanobiotique, la bionique ou le génie génétique.

Faire peur avec la cyborgisation dans sa version posthumaniste ${ }^{5}$ ou dans celles des genders studies est un procédé idéologique dont Dominique Lecourt a su démonter le mécanisme : que des imaginaires produisent des modifications représentationnelles sur l'incorporation des techniques a sans doute des

\footnotetext{
${ }^{1}$ MalabourC. (2009), Ontologie de l'accident, Essai sur la plasticité destructive, Paris, ed Leo Scheer.

${ }^{2}$ Yonnet P. (2006), Le recul de la mort. L'avènement de l'individu contemporain, Paris, Gallimard.

${ }^{3}$ Testart J. (2009), Cannibales et marchands à la recherche de l'embryon, Libération, 27 avril, p. 36.

${ }^{4}$ Le Douarin N. (2007), Les cellules souches, porteuses d'immortalité, Paris, O. Jacob.

${ }^{5}$ Besnier J.-M. (2009), Demain les post-humains, Paris, Hachette.
} 
conséquences, comme Jules Verne ou Mary Shelley ont pu le faire, sur l'invention technobiologique en définissant moins des programmes que des voies insensées trop souvent assimilés à de l'impensable sinon à de l'inhumain. La vigilance des sociologues et des moralistes agitent soit le catastrophisme soit la fin de l'homme. La crise serait aussi ontologique, l'être humain aurait perdu le contrôle

Face aux succès de ces thèses nous alarmant, une autre voie, il est vrai relevant d'un imaginaire du métissage ${ }^{6}$ est déjà à l'œuvre : celle de devenir hybride, c'est-à-dire de ne rester ni entièrement naturel ni de se réduire à un corps machine. Ce mélange techno-biologique, bionique utilise la matière biologique du corps pour y incorporer un morceau du corps d'un autre (greffe, gestation pour autrui) ou un dispositif technique (implants, prothèses): l'interaction est le paradigme de l'hybridité car la plasticité, la résilience et la recalibration exigent du corps vivant une technomorphose par un processus adaptatif dont le rejet immunologique ou mécanique sera l'épreuve et la preuve, selon.

\section{Une hybridation relationnelle}

L'hybridation, comme toute ontologie de la relation ${ }^{7}$, est relative au dispositif technique qui la produite et aux dispositions du corps qui l'accueille. Elle n'est pas idéale car elle implique une télésurveillance nouvelle forme du bio-pouvoir comme celle des RFID $^{8}$ et participe d'une nouvelle forme d'autocontrôle des populations biopolitisés par eux-mêmes. Elle n'est pas idéale, la défenestration de Gilles Deleuze suffirait à le montrer, car elle augmente la dépendance $\mathrm{du}$ corps biologique envers les dispositions de respiration, d'alimentation, de gestation et de motricité sans lesquelles la mort serait sans doute plus immédiate.

C'est moins une société postmortelle ${ }^{9}$ cette hybridation que la conséquence de la philosophie du dépassement de soi, ce que les gender et les queer studies ont accompli dans la notion de performativité du soi. S'il est vrai que la performance épuise le sujet ${ }^{10}$ jusqu'à la fatigue d'être soi ${ }^{11}$, cette psychopathologie de la souffrance identitaire ${ }^{12}$ ne doit pas consumer le sujet au point de lui interdire toute performativité créatrice. La performativité ne met pas le corps en miettes, elle est plutôt la conséquence de la dispersion épistémologique du corps.

\footnotetext{
${ }^{7}$ Glissant E. (2009), Philosophie de la relation, Paris, Gallimard, p. 84.

${ }^{8}$ Alberganti M. (2007), Sous l'oeil des puces : la RFID et la démocratie, Ed Acte Sud, p. 65.

${ }^{9}$ Lafontaine C. (2009), La société post-mortelle, Paris, Le Seuil, p. 75.

${ }^{10}$ Isabelle Queval,

${ }^{11}$ Alain Ehrenberg,

${ }^{12}$ Christophe Desjours
} 
Un exemple pour illustrer ce débat celui des mères porteuses. Il y a eu trois étapes avant d'arriver aux mères porteuses :

1. La dénaturalisation de la famille mononucléaire : recomposition des familles, divorces, etc.

2. Le traitement de la fertilité : avec l'insémination artificielle, les enfants sont produits indépendamment de la sexualité, de la parentalité ${ }^{13}$ biologique et de la distinction des familles.

3. L'apparition du concept de «gestatrice » : des femmes qui ne sont ni la mère biologique ni la mère symbolique de l'enfant (dans l'adoption, le parent est un parent symbolique).

N'y a-t-il pas une instrumentalisation du corps ? La loi sur le corps humain de 1994 interdit toute atteinte à la dignité de la personne, le corps d'une personne étant son corps, il ne peut être ni aliéné ni vendu ni légué ni donné, sauf dans un cas, qu'en cas de nécessité médicale pour la personne, ou à titre exceptionnelle dans l'intérêt thérapeutique d'autrui. Ces pratiques prouvent que le corps n'est pas interchangeable selon un principe d'insubstituabilité ${ }^{14}$.

Irène Thery ${ }^{15}$ défend la levée de l'anonymat dans l'IAD (Insémination avec Donneur) et dans l'AMP (Assistance Médicale à la Procréation) pour permettre au sujet d'avoir accès à son histoire avec la compréhension du désir de donner son matériau biologique : « A qui dois-je d'être né(e) ? ». Il faut éviter de transformer les «donneurs d'engendrement en fantômes anonymes » sans agiter « le spectre classique de la déroute des valeurs » selon une éthique du contradictoire.

Le corps n'est pas en miettes dès lors que l'hybridation technobiologique produit des métissages, des mixités et des mélanges qui ne sont ni des confusions de genre, ni le refus de repères symboliques. Devenir hybride ce n'est jamais l'être définitivement, la part de l'autre, Jean Luc Nancy l'a décrit comme celle de l'intrus) est incorporée en nous au point qu'une recomposition identitaire fragile et dynamique met le sujet au travail de son altérité qui pourrait l'altérer entièrement si l'équilibre interactif ne devait être maintenu par des protocoles contraignants et permanents comme dans la séropositivité, la greffe, les implants choléaires ou les prothèses des hanches...

\section{Quel sens au progrès technique?}

Le progrès technique n'est pas seulement «egotechnique ${ }^{16}$ car il incorpore autrui et ses procédures-dispositifs en nous nous rappelant matériellement qu'aucun corps humain n'est naturel et que nous sommes

\footnotetext{
${ }^{13}$ Flavigny C. (2009) Avis de tempête sur la famille, Paris, Albin Michel, p. 131-182.

${ }^{14}$ Agacinski S. (2009) Corps en miettes, Paris, Flammarion, p. 134.

${ }^{15}$ Thery A., Noizet A. (2009), Procréations assistées, secrets , accès aux origines, Esprit, Mai, La filiation saisi par la médecine, Paris, Seuil, p. 77-81.

${ }^{16}$ Stoterdjik P. (2005) Ecumes Sphères 3, Paris, Le livre de poche, p. 534.
} 
constitués par les autres en nous. Les cultural studies nous ont déjà démontré combien l'hybridation exigeait de dépasser le dualisme corps-technique et le critère de l'intégrité purifiée du soi pour s'apercevoir toujours déjà redevable d'un autre, nous provenons tous du dedans et de la matière du corps des autres.

Dans doute la tentation post-humaniste existe de purifier le corps des scories biologiques pour dire adieu au corps ${ }^{17}$, mais il serait injuste d'assimiler toutes les biotechnologies au seul argument heideggérien de l'arraisonnement de la nature, même dans la version stoterdjikienne d'un parc humain.

Comme le rappelle Alain Berthoz la simplexité du vivant ${ }^{18}$ n'est pas réductionniste ni réductible à une seule logique. Le vivant trouve des solutions pour hybrider le monde et les techniques qui lui sont proposées en les incorporant en nous de manière d'abord inconsciente avant que nous en formulions consciemment une philosophie de l'hybridité, par exemple. La critique du post-humanisme ne se fonde pas ici sur un néo-vitalisme: elle instaure l'interaction du corps avec ses techniques et les technologiques que la culture lui fait incorporer comme le milieu de sa matérialité. Judith Butler ${ }^{19}$ précise combien la représentation vient performer la matérialité du corps en assignant ce que doit être le sujet à un discours qui définirait sa place. L'intersex, les chimères, les greffés, les implémentés, les métisses, les pharmacodépendants, les prothésés.. sont inassignables ou alors, rappelle Guillaume Le Blanc ${ }^{20}$, invisibilisés socialement afin de renforcer les oppositions binaires.

L'hybridation $^{21}$ est une technique, d'abord mythologique puis scientifique, qui consiste à réunir deux corps ou parties ensemble pour obtenir un bien être, un mieux être ou un nouvel être, trois degrés qui conduisent de la perfectibilité au perfectionnement. En effet l'hybridation peut, en actualisant les vertus endogènes du vivant, utiliser de la perfectibilité ou plasticité d'un corps pour en faire exprimer toute la résilience et la recalibration.

Comme adaptation formative l'hybridation produit un bien être lorsqu'elle améliore sans guérir la fonctionnalité du corps vivant: en reculant l'handicapibilité corporelle, l'hybridation d'une prothèse ou d'un greffon à une fonctionnalité défaillante favorise une meilleure autonomie dès lors que l'incorporation de l'implant ne perturbe pas de trop les conditions subjectives de l'existence physique et psychique. Le sentiment d'intrusion et la sensation du membre fantôme témoignent combien la souffrance psychique et la douleur neurophysiologique peuvent conserver respectivement la dette de l'Autre et la trace du schéma corporel comme un vécu.

\footnotetext{
${ }^{17}$ Le Breton D. (1999), L'Adieu au corps, Paris, Métaillié, p. 14-15.

${ }^{18}$ Berthoz A. (2009) La simplexité, Paris ; O. Jacob.

${ }^{19}$ Butler J. (1993), Ces corps qui comptent. De la matérialité et des limites discursives du « sexe », trad.fr Charlotte Nordmann Paris, ed Amsterdam, 2009, p. 17.

${ }^{20}$ Le Blanc G. (2009), L'invisibilité sociale, Paris, P.U.F., p. 46.

${ }^{21}$ Andrieu B. ( 2008), Devenir hybride, Presse Universitaires de Nancy, Préface Stélarc.
} 
Comme amélioration somatique, l'hybridation instaure un mieux être recule l'amoindrissement de l'être en reculant les limites de la maladie et de la mort : cette délivrance du patient de la logique fatale du destin pour le temps incertain d'une dépendance à une machine qui paraît le maintenir dans la vie. Cet équilibre instable entre ce qu'apporte l'hybridation et ce qu'elle instrumentalise dans le sujet par une médicalisation de son existence est souvent compris comme une expropriation du corps. L'indéfinie amélioration transforme le mieux être en exigence de déshandicaper, définition d'une santé absolument exigible.

Comme création biotechnologique, le nouvel être hybride introduit dans l'environnement des modes inédits d'existence qui modifient l'exercice du vivant. Il abhorre, comme Iron Man ou Wolverine, des exosquelettes et des implants qui sont définissent une nouvelle identité tant dans le schéma corporel que dans l'image du corps.

\section{Une auto-santé bio-anthropologique}

L'expérience de santé ${ }^{22}$, le self-control et la conscience corporelle sont trois attitudes élaborées du cours de l'histoire de l'autosanté ${ }^{23}$ dont certaines techniques actuelles voudraient réunir en une seule pratique ${ }^{24}$. En soi-même le sujet se modifie par un travail réflexif sur sa posture ${ }^{25}$, sa respiration, son activité. Le corps devient son propre jardin ${ }^{26}$. Etre bien dans sa peau est devenu le nouvel impératif esthétique ${ }^{27}$. Mais sans une intériorisation la santé est certes visible mais si peu vécue.

Le mythe de l'externalisation dans la surface de la peau déride le corps vieux ${ }^{28}$ sans toujours être incorporé pour favoriser un changement par une prise de conscience. Mais l'appropriation de la santé par le vécu corporel ${ }^{29}$, si elle ne guérit pas obligatoirement, accompagne le patient dans son agentivité corporelle. La modification en douceur du corps ${ }^{30}$ exige une prise de conscience par le sujet des différents degrés d'autosanté qu'il veut s'appliquer selon son modèle et ses normes.

\footnotetext{
${ }^{22}$ Andrieu B. (2009), Sentir sa santé, dans Alexandre Klein ed., Les sensations de santé, P. U. de Nancy

${ }^{23}$ Andrieu B. (2008), L'auto-santé, dans B. Andrieu et G. Boetsch eds., Dictionnaire du corps, Paris, ed CNRS.

${ }^{24}$ Bligny Y., (2008), Automassage bioharmonique, Bernex, Jouvence.

25 " Retrouver son corps être en forme est une tâche qu'il faut mener au quotidien » dans Benjamin Ascher, 1998, La silhouette retrouvée. Les méthodes,les conseils, les traitements pour façonner son corps, Paris, L'Archipel, p. 224.

${ }^{26}$ Le livre est contre la cellulite. S.M. Braun, N. Corbasson, 1977, Vaincre la cellulite, ce mal de la solitude, Paris, Tchou, p. 31-44. Alain Mességué, 1980, Au jardin de ton corps, Paris, ed. Pastorelly.

${ }^{27}$ Face and Body, 2008, Marie Claire.

${ }^{28}$ Bloch D. Heilbrunn B., Le Gouès G. (2008), Représentations du corps vieux, Paris, P.U.F, p. 86.

${ }^{29}$ Comte-Sponville A. (2009), Du Corps, Paris, P.U.F., p. 34.

${ }^{30}$ Colinon M.C. (2009), Tatouage, Piercing, Modifier son corps en douceur, Paris, la Martinière, p. 14.
} 
Le vécu corporel de santé ouvre le sujet au langage social des émotions ${ }^{31}$ : le langage corporel implique un apprentissage à penser ${ }^{32}$ son corps, sa santé ou sa sexualité dès le plus jeune âge, à la condition que cette éducation ne conduise pas seulement à l'illusion d'un égo-détermination et ouvre la possibilité à une subjectivation réfléchie du soi.

Cette subjectivation repose sur le courage de la vérité : Selon Michel Foucault le médecin et le gymnasiarque " possèdent un savoir caractérisé comme tekhnê, savoir-faire, c'est-à-dire impliquant des connaissances, mais des connaissances prenant corps dans une pratique et impliquant, pour leur apprentissage, non seulement une connaissance théorique, mais tout un exercice (toute une askêsis ou toute une meletê) ${ }^{33}$; sans prise de corps par les exercices les connaissances médicales et physiques seraient un corpus théorique dont l'efficacité serait extérieure au sujet. L'auto-santé est possible quand le soi a luimême traversé, sinon les conditions de la maladie, du moins ceux de son exercice: celui-ci transforme par l'expérience l'enseignement jusqu'à la parrêsia, le dire vrai, qui « elle risque la mort de celui qui la pratique $»^{34}$

\section{Une égalité citoyenne face l'auto-santé?}

Des modes de subjectivation du sujet corporel contemporain dans la relation médicale à soi et au monde social contemporain sont présents dans l'expérience vécue explicite du corps vulnérable et du sujet ontologique. Ils sont un processus de subjectivation, d'auto réalisation de soi, l'auto désignation d'un soi corporel, une auto protection qui passent autant par l'auto soin que par l'auto santé ${ }^{35}$ : quelles sont alors les pratiques d'automédication des personnes vulnérables quant on doit tout de même se soigner? A partir de quels critères objectifs et subjectifs, et sous quelles injonctions sanitaires et sociales, ces populations passent-elles ou non de l'auto-médication à la consultation ? Comment le sujet vulnérable se représente-il dans ses pratiques d'autosanté et les actions des professionnels? Jusqu'où des visions du monde sanitaire et social biolégitiment-elles l'accès aux soins et à la protection sociale et des idéologies du corps sous-tendent. Comment se fabriquent les comportements

\footnotetext{
${ }^{31}$ Fernandez F., Lezé S., Marche H., eds. (2008), Le langage social des émotions. Etudes sur les rapports du corps et de la santé, Paris, ed Anthropos, p. 15.

${ }^{32}$ Béliste C. (2009), Photolangage Corps, communication et violence à l'adolescence : Apprendre à penser sa sexualité à l'adolescence - Construire des repères en groupe, Lyon, Ed Chronique sociale, p. 51.

${ }^{33}$ Foucault M. (1984), Le courage de la vérité, Paris, Seuil/Gallimard, 2009, p. 23.

${ }^{34}$ Op.cit., p. 24.

${ }^{35}$ Dans le rapport Coulomb (2007), l'auto-diagnostic, l'automédication sont devenues une pratique inégalitaire du pauvre, notamment dans la population défavorisée économiquement et socialement, boudée jusqu'alors en France, l'automédication a depuis peu gagné du terrain et représente $8 \%$ du marché pharmaceutique en valeur. Le rapport Toussaint rappelle, par ailleurs que l'étude des pratiques d'auto médication mises en œuvre par les populations vulnérables sont rendues nécessaires dans l'étude des pratiques de prévention, afin d'améliorer les dispositifs d'éducation pour la santé.
} 
individuels et collectifs d'auto médicalisation, d'auto protection des populations les plus vulnérables et leurs effets sur les pratiques corporelles d'autosanté ? .

Dans cette autosanté existe-t-il un autodiagnostic ? A quels réseaux de sanitaire ou social s'adresse-t-on? Quelle est l'influence des facteurs ethniques sur ces pratiques? ${ }^{36}$ C'est-à-dire quelles sont les interactions sociales que ces populations créent autour de la question de la santé et de protection du corps, comment élaborent-elles leur prise de décision ? La sous déclaration, ou le non recours à la prévention, au dépistage ou au réseau social implique t-elle une non pratique de santé ${ }^{37}$ Sur quoi repose cette sous déclaration? Est-ce un manque d'information d'accès à l'information, d'éducation à la santé, d'échec des professionnels à communiquer avec le vécu subjectif du vulnérable. Le non recours à des pratiques de dépistage implique til l'absence de pratiques de protection?

Aussi, le rapport de Mme Bérengère Poletti ${ }^{38}$ dresse-t-il d'abord un inventaire des perspectives offertes par les progrès scientifiques pour améliorer l'efficacité des aides techniques destinées à compenser les conséquences du handicap. Puis, il expose les obtsacles qui entravent la diffusion de ces aides et les moyens d'améliorer une situation qui n'est pas satisfaisante. Il souligne enfin la nécessité de renforcer le volet scientifique et technologique des politiques publiques mises en oeuvre en faveur des personnes handicapées et de favoriser un plus large accès à l'innovation.

Le rapport sur "les apports de la science et de la technologie à la compensation du handicap", commandé en octobre 2007 à la députée UMP des Ardennes, Bérangère Poletti et approuvé le mardi 2 juillet 2008 par les sénateurs et députés de l'OPECST, souligne les "faiblesses structurelles" de la recherche française dédiée au handicap. Mme Poletti a ainsi préconisé avec L'Office parlementaire d'évaluation des choix scientifiques et technologiques (OPECST), la mise en place d'une fondation pour la recherche sur la compensation du handicap, qui "financerait les recherches sur des produits innovants et aiderait les PME à les commercialiser", lors d'une conférence de presse. Car le marché français des aides techniques au handicap fonctionne mal, avec "très peu de constructeurs" et une "étroitesse du marché" qui "interdit la mise en oeuvre d'une réelle concurrence" qui agirait sur les prix.

\footnotetext{
${ }^{36}$ Notre recherche ne peut ignorer le rapport sur la prise en charge des médico-psycho-sociale des migrants et étrangers en situation précaire, paru en 2007 , Editions le Comede www.comede.org

${ }^{37}$ Les personnes aux revenus les plus faibles se perçoivent en moins bonne santé que le reste de la population. Si elles déclarent moins fréquemment certaines maladies courantes, comme celles des yeux, elles sont en revanche plus nombreuses, adultes comme enfants, à souffrir de certaines pathologies comme les maladies de l'appareil digestif ; $11 \%$ des plus pauvres souffrent de caries contre $6 \%$ du reste de la population. Elles vont par ailleurs moins souvent chez le médecin, surtout chez les spécialistes. Elles sont également moins bien couvertes par la protection sociale : $22 \%$ d'entre elles n'ont pas de complémentaire santé contre $7 \%$ du reste de la population. Enfin, la prévention et le dépistage sont des pratiques de protection beaucoup moins répandues parmi les personnes les plus pauvres, contribuant à creuser encore l'écart entre elles et le reste de la population.( Etude INSEE, octobre 2007)

${ }^{38}$ Poletti B. (2008) Les apports de la science et de la technologie à la compensation du handicap, Paris, Sénat.
} 
Pour l'Office, il est "nécessaire" de "simplifier les procédures de mises sur le marché". Et le Conseil de la concurrence doit être saisi pour "vérifier s'il existe des pratiques anti-concurrentielles" dans ce secteur alors que le prix d'un même produit peut varier du simple au triple entre les Etats-Unis et la France. De plus, "beaucoup de produits récents ne sont pas intégrés dans la politique de remboursement", regrette Mme Poletti. Un crédit d'impôt serait "souhaitable" pour financer une partie de l'aide technique pour les personnes handicapées à plus de 50\%, propose l'Office. Le rapport concentre par ailleurs les espoirs suscités par les nouvelles technologies, souvent venues de l'étranger, comme le contrôle direct d'une machine par le cerveau, des fauteuils roulants équipés de bras robotisés ou des "exosquelettes" pouvant être fixés sur les membres et les faire bouger. Pour Mme Poletti, "l'amélioration de l'interface entre l'homme et la machine constitue une vraie révolution pour les handicapés les plus lourds".

\section{Conclusion}

Vivre sans frontières, composé d'une double identité, être un soi sans mêmeité, avoir un corps impropre n'interdisent pas à l'hybride d'avoir une identité temporelle et spatiale. Là où chacun espère de la clôture virtualisée éviter l'épreuve physique du monde, les hybrides transportent en eux la part des autres en y recomposant sans cesse leur identité. L'hybridité interactive qui constitue notre soi est occultée par l'égologie dans ses limites pathologiques et libérales. A la fatigue d'être soi peut succéder, au delà de l'ivresse de la découverte d'autrui, l'invasion des autres en nous.

L'altération est le risque principal de l'hybridation faute de contrôler, malgré la technique, les conséquences de l'incorporation. Car l'hybridation est biosubjective et non seulement mécanique. Aux conséquences physiologiques comme la reprogrammation du schéma corporel chez le greffé ou le prothésé dans le membre fantôme, s'ajoutent les conséquences sociopolitiques d'un nouveau lien social : celui de la mixité sociale.

Les autres ne sont pas hors de nous, ils sont dans la mosaïque de nousmêmes un fragment dont la composition forme le style de notre existence. 